\title{
Science Unlimited?
}

\author{
Carl G. HempeL* \\ Princeton University
}

\section{Introduction: the problem}

Our age is often called an age of science, and with good reason: the advances made during the past few centuries by the natural sciences and more recently by psychology and the social sciences have vastly broadened our knowledge and deepened our understanding of the world we live in and of our fellow men; and the basic soundness of the insights achieved by science is eloquently attested to by the striking success of their applications, both constructive and destructive, which have radically changed the quality of life on our planet and have left their characteristic imprint on every aspect of contemporary civilization.

Contemplating the increasingly rapid expansion of scientific knowledge, one may well wonder how far this process can continue; whether all questions about the world can ultimately be answered by scientific inquiry, or whether there are absolute limits beyond which scientific knowledge and understanding can never go. This is the problem to be considered in my paper.

My discussion is meant to apply to all branches of scientific and scholarly inquiry, from the physical sciences through biology and psychology to the social and historical disciplines; thus, when the terms 'science' or 'scientific' are used, they are meant to refer comprehensively to all of empirical science. I shall be concerned, however, only with pure, or basic, research and not with the technological applications of scientific knowledge or procedure; accordingly, I shall not discuss the limits of the technologically attainable control of empirical phenomena.

Pure scientific research has two principal objectives, which I will briefly call knowledge and understanding: science seeks to ascertain what goes on in this world of ours, and to understand why. I propose to consider the question of limits first with respect to the pursuit of scientific knowledge and then with respect to the quest for scientific understanding.

It will help further to circumscribe our central topic if we acknowledge at the outset one limitation that is shared by scientific knowledge and by scientific understanding. Obviously, there are many questions of empirical fact and many problems of explanation which science is unable to answer at present. Moreover, since the life span of humanity is presumably finite, scientific inquiry as carried out by human beings will never raise, let alone answer, more than finitely many questions of fact and of explanation, whereas the set of all logically possible such

\footnotetext{
* This article is a result of work supported by a research grant from the National Science
} Foundation, Washington, C. C. 
questions is infinite. Hence, infinitely many questions aimed at knowledge or understanding will, in fact, never be answered by science.

But this limitation is, if I may say so, only of a practical kind; it does not bear on the philosophically interesting problem whether scientific knowledge and understanding are limited "in principle", i.e., in ways that would remain unaffected even if scientific research could go on indefinitely. Such limits-in-principle would have to stem from certain general logical and methodological characteristics of scientific knowledge and understanding. It is with the question of such limits that we will now be concerned.

\section{The inductive uncertainty of scientific knowledge}

There is one pervasive characteristic of scientific knowledge that may well appear to constitute an insuperable limitation, namely, its inductive uncertainty. Consider any of the statements that purport to express scientific knowledge, such as Galileo's law of free fall or Kepler's laws of planetary motion or contemporary theories in the natural and the social sciences, or statements about particular facts, such as that the Moon has no atmosphere, or that King George III of England suffered from porphyria: on what grounds can they be claimed to express knowledge, and thus, to be true? How does empirical science certify its knowledge claims?

As is well known, the answer - or at least an important part of the answer is that the statements asserted by science are ultimately certified by reference to relevant empirical "data", i.e., evidence obtained by experimentation, by systematic observation, by sociological field work, by the careful scrutiny of historical records or relics, and so forth. What can such evidence show concerning the truth of the statement, or "hypothesis", under scrutiny?

Let us assume, for the sake of the argument, that there are no errors or uncertainties about the recording of the evidence, so that the "data sentences" or "evidence sentences" describing the evidence for a given scientific assertion are all true. Even then, the evidence can show at best that in the particular cases thus far examined, the experimental or observational data were in accord with the hypothesis under investigation. But that does not suffice to guarantee the truth of the hypothesis. Galileo's law, for example, concerns not only those cases of free fall which have been scientifically examined so far; it makes a general assertion about the relation between elapsed time and distance covered in any case of free fall, including the fall of prehistoric meteorites that flashed across the sky unseen and unexamined by any human observer, as well as all cases of free fall that are as yet in the future. And clearly, however many instances of free fall may have been observed and found to conform to Galileo's law, they afford no guarantee that all the unexamined cases did or do or will equally conform to that law. 
This consideration can be generalized. No matter how extensively an empirical hypothesis has been tested, and no matter how well it has been borne out by the test findings, it may yet fail in cases that have not been examined. The supporting evidence does not suffice to verify the hypothesis, i.e., to establish its truth with deductive certainty; it can only lend the hypothesis more or less strong "inductive support".

Can this inductive uncertainty properly be regarded as a shortcoming of scientific knowledge? Such criticism would reflect the view that the quest for knowledge is a quest for certainty, and that scientific ways of establishing empirical claims are essentially defective because they fall short of this ideal of certainty. But where does this ideal stem from, and can it ever be attained? If there is any field in which such certainty is possible, it surely is the non-empirical field of pure mathematics.

Let us consider briefly the proverbial certainty of mathematical propositions such as the theorems of Euclidean geometry. These propositions are established by mathematical proof, which shows that they follow logically from the axioms of Euclidean geometry; so that if the postulates are true, the theorems are certain to be true as well. (More explicitly: for any interpretation of the primitive geometrical terms that makes the postulates true, the theorems are true as well.) The certainty of the theorems is thus relative to the postulates. And indeed, in a non-Euclidean geometry, some of the Euclidean theorems do not hold: they are not certain relative to the postulates in question; i.e., they do not follow deductively from the latter.

But if a sentence $T$ follows logically from certain other sentences $P_{1}, P_{2}, \cdots$, $P_{n}$ - let us call their conjunction $P$ for short - then $T$ simply restates part of what is asserted by $P$. For if $P$ logically implies $T$, then, as is readily verified, $P$ is logically equivalent to the conjunction of $T$ and the conditional sentence $T \supset P$; or briefly:

$$
\text { If } P \rightarrow T \text {, then } P \leftrightarrow T \cdot(T \supset P)
$$

The certainty of $T$ relative to $P$ is thus purchased at the price that $T$ asserts nothing that is not implicitly asserted already by $P$. If a statement is to be certain relative to the grounds by which it is supported, then its content cannot go beyond that of the statements expressing those grounds.

But the content of a hypothesis in empirical science does go far beyond the content of the data sentences adduced in its support. Scientific laws, for example, while supported by statements about instances examined in the past, make assertions about countless other, unexamined, instances. Indeed, it is an important objective of science to arrive at general laws and theories that yield predictions concerning future events and retrodictions concerning unexamined past ones: and such laws and theories evidently assert more than the data sentences by which 
they are supported. Precisely because science seeks knowledge that reaches far beyond the supporting evidence, its empirical claims cannot consistently be required or expected to be certain relative to that evidence; the ideal of empirical knowledge with certainty is logically self-contradictory. And surely, science cannot be held to be limited because of its failure to meet a standard of perfection which is logically inconsistent.

Let us briefly consider the issue from a somewhat different vantage point. Empirical science, we noted, supports its knowledge claims by means of evidence obtained by careful empirical tests. Could the acknowledged fallibility of this procedure be adjuged a limitation on the ground that there are, or might be, superior alternative methods that would yield knowledge-with-certainty? Perhaps, such knowledge could be attained by a gifted clairvoyant or by the use of procedures not tied to the requirements of empirical evidence?

Such sources may well yield some true statements, as may indeed blind guessing. Suppose that two persons consider the sentences

(1) There will be a manned landing on Mars before the year 2000

(2) There will be no manned landing on Mars before the year 2000, and suppose that one of them, by simply guessing or flipping a coin, selects the first sentence and asserts it, while, by the same method, the second person is led to select and assert the second sentence. Then it is certain that one of them has asserted a true statement.

But the crucial question in our context is not, of course, whether some alternative to the method of empirical testing may yield true statements, but rather whether it can be known with certainty that all statements obtained by that method are true. And the answer to that question is: no; for the certainty in question is not, of course, to consist in a subjective sense of confidence or conviction, but will have to be objectively guaranteed by supporting grounds. But whatever data we may have concerning the effectiveness of the method in question, they will concern past instances of its application; and, for reasons previously considered, these do not suffice to ensure the truth of the statements the method may yield in future applications. The claim concerning the superior effectiveness of alternative methods is itself subject to inductive uncertainty; no alternative to the procedures of empirical science can give us factual knowledge with certainty.

\section{On limits of the potential scope of scientific knowledge}

From the issue of limits to the reliability of scientific knowledge claims let us now turn to the subject of limits to the scope of scientific knowledge. Are there any questions of fact that are in principle beyond the reach of a scientific answer?

In a book published some years ago that was intended to deflate various 
claims made for the power and efficacy of science, there occurred an argument that science cannot disprove the existence of ghosts since to point out that no scientist had ever observed a ghost does not suffice to show there are none; for ghosts might be allergic to scientists and refrain from appearing in their presence. Such reasoning evidently rests on a too simple-minded view of scientific testing. The hypothesis that there are ghosts who do appear, but only in the absence of scientists, might well be indirectly testable by means of suitable automatic recording instruments. Suppose we press the argument further by offering the hypothesis that there are ghosts who do appear, but only in the absence of scientists and scientific instruments. Even this might still be testable, for example by observers or recording equipment stationed at some distance, or by later examination of the scene itself, which might show traces of ghostly appearances.

To make it impossible in principle for science to find out about the existence of our ghosts, we would have to propound an even more fanciful proposition, namely: There are ghosts whose existence cannot be ascertained by any observer or any test equipment; they produce no effects which even in the most indirect manner manifest themselves in anything accessible to human experience.

But this is tantamount to saying that there are ghosts, but everything in the world of our actual and potential experience proceeds exactly as if there were none. And this sentence makes no empirical assertion at all because for a significant empirical claim, it must be possible to indicate what it asserts, however indirectly, about some ascertainable aspect of the world of our experience; it must be possible to specify what kinds of experiential data would lend it support, what others would tend to disconfirm it. Since, then, the given sentence makes no empirical assertion, the fact that scientific inquiry can neither establish nor disconfirm it does not reflect any limit of the potential scope of scientific knowledge.

Let me put the point a bit differently: Granting that scientific inquirty can give us no knowledge concerning those ghosts, is there any other source of insight that can? On what grounds could such claims to knowledge be based? Not on empirical evidence, since our fanciful hypothsis specifically precludes all empirical manifestations. Nor could the claim be based on premonitions or other intimations experienced, perhaps, by individuals endowed with a special sensitivity: for reports by "psychic" individuals about such intimations would constitute indirect evidence concerning those ghosts. Nor, of course, can the ghost-hypothesis be known to be true on purely logical or conceptual grounds. Hence, it is quite unclear what could even be meant by "knowing" whether the hypothesis is true; hence, the case surely reveals no limits of potential scientific knowledge.

\section{Scientific explanation: general characteristics}

I now turn to the second of my two principal topics, namely, the limits of 
scientific understanding.

The understanding of empirical phenomena that science can give us is conveyed by scientific explanations. Broadly speaking, science explains why a given event came about by showing that it occurred in certain particular circumstances (in the natural sciences often called initial and boundary conditions) in accordance with certain general laws of nature or well-established theoretical principles. For example, an explanation of a rainbow occurring on a particular occasion may note, first certain particular conditions obtaining at the time, especially the presence of spherical water drops suspended in the atmosphere and of sunlight impinging on them; secondly, it shows that, as a consequence of the laws of optics, rays of sunlight are refracted and reflected in the drops in such a way as to produce the characteristic rainbow pattern. Thus, the phenomenon is explained by showing that, under the given particular conditions, it "had to" occur according to the specified laws. The explanatory account can accordingly be conceived as a deductive argument whose premisses - jointly referred to as the explanans - consist of the relevant laws and of descriptions of the particular circumstances, while the conclusion, the so-called explanandum sentence, describes the phenomenon to be explained. The argument enables us to understand the phenomenon by showing that, given the laws and the particular circumstances, its occurrence "was to be expected" in the sense that it could have been inferred from the explanatory information.

Scientific explanation is not restricted to individual events, however, such as the appearance of a rainbow or of a lunar eclipse on a particular occasion; science also seeks to explain the general uniformities expressed by the laws of nature. This is usually done by showing that they hold in virtue of a well-established comprehensive theory from which they can be derived, as when the uniformities expressed by the laws of refraction and reflection are explained by means of the electromagnetic wave theory of light.

In the cases considered so far, the explanatory laws or theoretical principles are of strictly universal form; they make a claim of the type: Whenever and wherever conditions of kind $F$ are realized, an occurrence of kind $G$ takes place. There is another mode of explanation, which relies on laws of a probabilisticstatistical form: Under circumstances of kind $F$, the probability that an occurrence of kind $G$ will take place is $r$. Explanations by such laws have come to play an increasingly important role in science, for example, in statistical mechanics and in the theory of radioactive decay. An explanation of this kind cannot show, of course, that the phenomenon to be explained "had to" occur in the sense that the explanandum sentence describing it is deducible from the explanans and thus must be true if the explanans is true. Rather, a probabilistic-statistical explanation, such as Boltzmann's statistical explanation of the ireversibility of certain 
physical processes, can show only that, given the information provided by the explanans, the occurrence of the explanandum phenomenon has a high probability. ${ }^{1}$

In dealing with the question of limitations of scientific understanding, let us distinguish, much as in our discussion of scientific knowledge, two different issues: first, the question of irremediable shortcomings of the kind of understanding that scientific explanation can afford; second, the question whether the scope of scientific explanation is limited in the sense that some aspects of our world are inaccessible in principle to scientific understanding.

\section{How vs. why}

It is sometimes said, by scientists as well as by philosophers, that science can at best tell us how empirical phenomena come about, but can never explain why. Unfortunately, proponents of this view do not say clearly what they take to be the difference between showing how and explaining why; they do not indicate what kind of an account would afford a genuine explanation-why, and by what means such explanations might be achieved if science cannot provide them.

In some cases, the view is associated with the idea that a proper explanationwhy requires resort to trans-scientific conceptions. Thus, it has been claimed ${ }^{2}$ that a genuine understanding of gravitational attraction is afforded by the conception that the attraction is a manifestation of certain "appetites or natural tendencies" closely related to love, which are inherent in physical bodies and make their "natural movements intelligible and possible". But the kind of affinity called love differs from gravitational attraction in many respects: It is not the case that everybody loves everybody else, nor does one's love for another regularly increase with the loved one's mass, nor does it decrease rapidly with distance. A defender of the idea might reply that these objections take the kinship between love and gravitational affinity too literally; that gravitational affinity should be conceived as a tendency of physical bodies to attract each other in the manner specified by the law of gravitation. But that all physical bodies have that tendency is asserted by the law of gravitation itself; and to call it a natural appetite akin to love is not to explain gravitational attraction, but to re-describe it in terms of an anthropomorphic metaphor. Such re-description may give us an enhanced sense of familiarity with gravitation, but the impression that we thus gain an understanding of the phenomenon is entirely deceptive.

1 The conception here outline of scientific explanation is presented in detail in my essay, "Aspects of Scientific Explanation", in C.G. Hempel, Aspects of Scientific Explanation and other Essays in the Philosophy of Science. New York: The Free Press; London: Collier-MacMillan Lmited, 1965; paperback edition, 1970; pp. 331505.

2 See J.F. O'Brien, "Gravity and Love as Unifying Principles", The Thomist, vol. 21 (1958), pp. 184-193. 


\section{Explanatory incompleteness}

Another charge of deficiency sometimes brought against scientific explanation is considerably clearer and more substantial. I shall call it the charge of essential incompleteness. In response to the question "Why does this phenomenon occur?", an explanation typically cites certain general laws and particular circumstances and shows that in view of these, the phenomenon "had to" occur. The explanatory facts adduced are simply stated but not explained in turn. For example, the explanation of a particular occurrence of a rainbow tells us that there is sunlight impinging on water droplets and that light rays conform to the laws of refraction and reflection, but it does not tell us why this is so. A scientific explanation in thus always incomplete in the sense that the explanatory facts it adduces are left unexplained and thus ununderstood. It may even seem that, as a consequence, an explanation in science never does more than reduce the problem of explaining one fact to the problem of explaining several others.

One facet of the view that the incompleteness here considered sets insuperable limits to scientific understanding was presented by the German physiologist Emil Du Bois-Reymond in his famous address "Ueber die Grenzen der Naturerkennens"." $\mathrm{Du}$ Bois-Reymond's argument in this case, and in the case of another problem soon to be considered, was aimed at showing that the problem in question could not be solved even in the most perfect state of scientific knowledge.

In Du-Bois-Reymond's view, all scientific understanding of nature amounts to reducing the changes occurring in the physical world to the motions of atoms as performed under the influence of central forces, such as gravitational attraction, which they exert upon each other. The most perfect state of scientific understanding would then be represented by what he calls "Laplace's Spirit", i.e. that superhuman intelligence imagined by Laplace in his characterization of mechanistic determinism. That superscientist would know all the force laws and would have combined them into one single universal formula; he would also be a super-observer, capable of ascertaining, at any moment, the state of the universe as represented by the masses, positions, and velocities, of all atoms at that moment; and, from the momentary state thus ascertained, he would be able to infer, by means of the universal formula, the state of the universe at any other time. To this Spirit, as Laplace puts it, nothing would be uncertain; both past and future would be present before his eyes. Du Bois-Reymond notes that such "astronomical knowledge" of material systems, as he calls it, is an ideal that human science

s This lecture, delivered in 1872, is reprinted in Emil Du Bois-Reymond, Reden, edited by Estelle Du Bois-Reymond. Leipzig: Veit und Co., 2nd ed., in two volumes, 1912; vol. I, pp. 441-473. Page references are to this reprinting, and quoted passages are translated from it. A somewhat abridged English translation, which is incorrect in some places, however, appeared in The Popular Science Monthly, vol. 5 (1874), pp. 17-32. 
can never fully attain; but he adds that there are certain problems which remain unsolvable even for the Laplacean Spirit, and which are thus unsolvable in principle by science. The Spirit, for example, would be able to infer "the original state" of the universe: but if he should find matter in that state to be at rest and unevenly distributed, he would not know whence the uneven distribution; if he should find matter in the original state to be in motion, he would not be able to account for the latter. ${ }^{4}$

Max Weber makes a similar point in commenting on the limited understanding affortded by the "astronomical knowledge" that Du Bois-Beymond and many other scientists of his time regarded as the ideal of scientific attainment. Speaking of the explanation, by general laws, of the state of a system at a given time by reference to an earlier state, Weber remarks: "Every individual constellation which it 'explains' $\cdots$ is causally explicable only as the consequence of another equally individual constellation which has preceded it. As far back as we may go into the grey mist of the far-off past, the reality to which the laws apply always remains equally individual, equally undeducible from laws." 5

Both Du Bois-Reymond and Weber limit themselves to pointing out that the scientific explanation of particular states or events requires reference to other particular states or events, which are left unexplained. As we have noted, explanatory incompleteness can be argued also on the ground that the general laws invoked are left unexplained.

Does the incompleteness here acknowledged mark a limitation of the understanding attainable through scientific explanation?

When the appearance of a rainbow on a particular occasion is explained in the way mentioned earlier, the explanation answers perfectly well the question to which it is addressed, namely, "Why is there a rainbow over there?" The question plainly does not ask why there are water droplets suspended in the air or why sunlight conforms to the laws of refraction and reflection. To be sure, the explanation may evoke such further questions in the mind of the person to whom it is addressed; but this is a psychological matter, and the problems thus raised are new explanatory problems.

For much the same reasons, an explanation does not in any logical or systematic sense "reduce" the problem of accounting for the original explanandum to that of accounting for the explanatory facts adduced. If, for example, we wish to explain a particular event as having resulted by law from certain other particular events, we have to show that those other events did take place and that the laws invoked do hold, but we need not show why.

- Du Bois-Reymond, loc. cit., pp. 449-450.

- Max Weber, On the Methodology of the Social Sciences, translated and edited by E.A. Shils and H.A. Finch. Glencoe, Illinois: The Free Press, 1949; p. 73 (italics quoted). 
Perhaps these remarks still leave the impression that scientific explanation is essentially defective because it falls short of the ideal of conveying a complete understanding of its subject - an understanding that would be afforded only by an explanatory account that explained every fact it asserted, thus answering every explanatory problem that can be raised within its context. But is such completeness a reasonable standard for appraising the adequacy of a mode of explanation?

If an explanation $E$ explains a given phenomenon, as well as the facts it cites for the purpose, as well as the facts cited to explain the latter, and so forth, then it clearly involves an infinite regress, and the total set $A_{E}$ of statements it contains will be infinite. This in itself raises questions about the appropriateness of the ideal of completeness, for we will normally require of an explanation that it be expressed in a finite number of statements.

A more serious objection against the ideal of completeness is this: Each of the non-empty subsets of the set $A_{E}$ of statements contained in an explanation $E$ will represent a "fact asserted by $E$ ". Completeness would require that for each of those subsets, $B$, there be another subset, say $C$, of $A_{E}$ which provides an explanation for the fact represented by $B$. But one of the subsets $B$ of $A_{E}$ is $A_{E}$ itself; and for it, $A_{E}$ cannot contain an explanation because what $A_{E}$ asserts cannot be explained simply by re-asserting $A_{E}$. No empirical fact as described by some set of sentences can be explained by means of the same sentences; no account of the type ' $p$ because $p$ ' is an explanation.

The argument just outlined clearly does not depend on the assumption that the explanations in question are all of the "covering-law" type. It applies equally to any presumptive mode of explanation in which the fact to be explained as well as the explanatory answer is expressed in the form of sentences - no matter how the relationship between explanans sentences and explanandum sentence is conceived, and no matter whether the explanation is couched scientific, metaphysical, or religious terms. No conceptualized account can explain anything without asserting something that it does not explain.

It is a fundamental misconception, therefore, to appeal to the ideal of completeness on the ground that "ultimate explanation must be possible in theory; otherwise, even partial explanation must fail" ; hence, failure to meet this ideal cannot count as a shortcoming or a limitation of scientific understanding.

\section{Riddles of explanation}

Now let us turn to the question whether the scope of scientific understanding is limited, whether there are any aspects of the world that are in principle beyond

- Ronald W. Hepburn, "Cosmological Argument for the Existence of God" The Encyclopedia of Philosophy (Paul Edwards, Editor in Chief). New York: The MacMillan Co. and The Free Press, 1967; vol. 2, pp. 232-237; quote from p. 236. 
the reach of scientific explanation.

An emphatically affirmative answer was put forward by Du Bois-Reymond in the lecture mentioned earlier. Du Bois-Reymond here distinguishes two kinds of unsolved scientific problems. Those of the first kind, which he calls riddles of the material world, are questions which, though not answered at the time, are answerable in principle and may well yield to further scientific inquiry. Those of the second kind, on the other hand, which he calls transcendent riddles, are incapable in principle of a solution by scientific means. One of the problems which he assigns to this class is the relation between mind and matter, or, more specifically, the question why it is that certain configurations in the human brain give rise to consciousness, thought, feeling, and other mental phenomena. As in the case considered before, Du Bois-Reymond refers to the Laplacean Spirit and argues that even this super-scientist with his astronomical knowledge of the brain would be able to establish no more than that certain mental states or events are associated with certain atomic configurations or events in the brain; the question why this is so would evidently be left entirely unanswered; hence it constitutes a transcendent riddle.

$\mathrm{Du}$ Bois-Reymond stresses the difference between this absolutely unsolvable riddle and such problems as the determination of the brain-mechanical processes associated with the performance of an arithmetical computation, which are far beyond the reach of science at present, but which might conceivably be solved by future research. In regard to such riddles of the material world, he remarks, the scientist has long been used, in manly resignation, to acknowledge: Ignoramus - we do not known, at least as yet; but in regard to transcendent riddles, like that of the of mind matter, the scientist has to decide upon a verdict much more difficult to render: Ignorabimus - we shall never know. ${ }^{7}$

A strikingly similar argument had been presented a few years earlier by John Tyndall in his address "Scientific Materialism", delivered in 1868 before the British Association for the Advancement of Science. ${ }^{8}$ Tyndall remarks that if the powers of our senses and our minds were vastly strengthened, we might be able to discover that "the consciousness of love ... [is] associated with a right-hand spiral motion of the molecules of the brain, and the consciousness of hate with a left-hand spiral motion": we would then know that this is so, "but the "WHY?" would remain as unanswerable as before." He concludes: "The problem of the connection between body and soul is as insoluble, in its modern form, as it was

7 Du Bois-Reymond, loc. cit., p. 464.

s Reprinted in John Tyndall, Fragments of Science, Westminster edition, 2 volumes. New York and London: D. Appleton and Company; no date imprint (1900?), pp. 75-89.

- Tyndall, loc. cit., p. 87. 
in the prescientific ages."10 "Science is mute in reply to these questions", he acknowledges; and, faithful to his scientific materialism, he extends this verdict to the competition: "But ... who else is prepared with a solution? ... Let us lower our heads, and acknowledge our ignorance, priest and philosopher, one and all."11

Although Du Bois-Reymond's narrowly mechanistic view of scientific explanation has long since been abandoned, the psycho-physical problem has continued to be widely regarded as inaccessible to a scientific solution. In a presidential address delivered before the British Association for the Advancement of Science almost 100 years after Tyndall's lecture, ${ }^{12}$ the chemist Sir Cyril Hinshelwood remarks that despite the great advances made by molecular biology, it "remains utterly incomprehensible.. how and why the brain becomes the vehicle of consciousness."13 Hinshelwood sees here an "inscrutable mystery" that is probably indicative of "the inherent limitations of human understanding."14

Is this view sound? Are the supporting arguments compelling? Let us note first that psychological phenomena are often explained by reference to physical occurrences, on the basis of suitable connecting laws; as when certain hallucinatory states are explained as brought on by drugs or by sensory deprivation, loss of consciousness by an extreme lowering of the blood sugar level, the occurrence of afterimages by certain kinds of optical stimulation, and so forth. The covering laws invoked in these cases are basically of this type:

At any time $t$ when an organism $x$ is exposed to physical conditions of kind $P$, then psychological, or mental, phenomena of kind $M$ occur:

\section{$(x)(t)(P x t) M x t)$}

Adherents of the view expressed by Tyndall, Du Bois-Reymond, and Hinshelwood would no doubt insist that the unsolvable riddle remains totally unaffected by these remarks; that there is no possibility of explaining why it is that physical occurrence of kind $P$ are generally associated with mental phenomena of kind $M$. But even this claim is debatable. For some specific psychophysical connections can certainly be explained at least in a modest fashion, namely, by subsumption under other, more comprehensive, psychophysical connections. Consider, for example, the law that ingestion of phenobarbital induces drowsiness. The psychophysical connection it asserts can be explained by pointing out that phenobarbital is a barbiturate, that all barbiturates are depressants of the central

10 Tyndall, loc. cit., p. 58.

11 Tyndall, loc. cit., p. 88.

12 Cyril Hinshelwood, "Science and Scientists", Bulletin of the Atomic Scientists, vol. 23, No. 6 (June 1967), pp. 30-36. (The article is an adaptation of the presidential address, delivered in 1965).

13 Hinshelwood, loc. cit., p. 35.

14 Hinshelwood, loc. cit., p. 36. 
nervous system, and that depression of the central nervous system is accompanied by drowsiness.

Without doubt, the discovery of such more comprehensive psychophysical laws is generally considered as advancing our understanding of the hypnotic effect of phenobarbital. But the objectors would argue that such discoveries do not bring us one whit closer to the solution of the basic riddle; for at every stage of the process, certain general psychophysical connections are invoked, and we never come to understand why certain purely physical conditions can give rise to mental states or events.

This problem is analogous, it seems to me, to that of explaining why one sort of physical occurrence, such as movement with friction, gives rise to a qualitatively quite different one, such as heat. In physics such connections are often explained by suitable theories. For example, the kinetic theory explains the generation of heat by friction by pointing out that heating a body is tantamount to increasing the kinetic energy of the random motion of its molecules, and that friction increases that energy for the molecules at the interface of the bodies concerned. Similarly, the theory of electromagnetic fields may be said to explain the connection between the flow of an electric current and the qualitatively quite different movement of a compass needle in the vicinity.

To understand why certain kinds of physical occurrence are associated with certain mental phenomena, we would then have to look for a theory exhibiting the connecting linkage, as it were.

It has often been argued in the philosophical literature that mental states and events should simply be regarded as indentical with the corresponding brain states or events, much as in the kinetic theory, the temperature of a body is identified with the mean kinetic energy of the random motions of its molecules. But a theory effecting such a linkage by identification would hardly satisfy the proponents of our riddle. For the identification of a mental state $M$ with a physical state $P_{M}$ involves the establishment of a general law to the effect that $M$ occurs when and only when $P_{M}$ does; and in regard to this law, the mind-matter riddle can be raised again.

A theory suited to answer the riddle would presumably have to employ a new set of basic concepts, which could not be qualified as strictly physical nor as strictly psychological; and the fundamental laws of the theory would have to link those new basic concepts to each other as well as to psychological concepts on the one hand and physical concepts on the other, in a manner that would permit the derivation of specific psychophysical connections. Such a theory would then explain those connections by subsuming them under theoretical laws which themselves are not of a psychophysical kind.

I do not know whether a unifying psychophysical theory of this kind will 
ever be developed, but the arguments offered by Du Bois-Reymond and others certainly do not preclude that possibility.

As one further potential candidate for the status of a transcendent riddle, let us briefly consider a question that has been raised by various philosophers, and that seems both profound and utterly unanswerable by scientific means: Why is there anything at all and not rather nothing?

A literal-minded defender of science might argue that science can explain, for example, why there is ice on the pond after a cold night, and that to show why there is ice on the pond is ipso facto to show why there is something. But this answer seems to miss the point of the riddle; for the explanation mentioned accounts for the presence of ice at a certain place by reference to the earlier presence there of water. The explanation thus already assumes that there is something rather than nothing, and no light seems to have been shed on the riddle.

But what kind of an answer would be appropriate? What seems to be wanted is an explanatory account which does not assume the existence of something or other. But such an account, I would submit, is a logical impossibility. For generally, when the question "Why is it the case that $A$ ?" is answered by "Because $B$ is the case", then surely that answer ean be adequate only if the assertion that $B$ is the case affords good grounds for the assertion that $A$ is the case, so that it is rational to say: If $B$ is the case, then indeed it is to be expected that $A$ is the case. This clearly is a necessary, though not a sufficient, condition for the adequacy of any explanation. ${ }^{15}$

But an answer to our riddle which made no assumptions about the existence of anything cannot possibly provide grounds for saying: It is to be expected that there is something rather than nothing. The riddle has been constructed in a manner that makes an answer logically impossible: and scientific explanation can hardly be held to be limited because it cannot satisfy a logically inconsistent requirement. Nor, indeed, can any other discipline or source of insight provide an answer to the riddle; an answer in terms of a prima causa, for example, presupposes the existence of that agency and thus the existence of something. No theory, no conceptual scheme, can explain the existence of anything without assuming the existence of something.

\section{Conclusion}

We have considered a variety of considerations which appeared to prove that scientific knowledge and understanding are flawed by insuperable defects and limitations. I have tried to show that those arguments fall short of their mark.

15 For a fuller discussion of this condition of adequacy, see Hempel, loc. cit., pp. 367368. 
While my critical comments have been directed explicitly only against the specific limitation claims here considered, I think they can be extended to any other arguments purporting to show that certain problems of knowledge or understanding concerning empirical subject matter are incapable in principle of a scientific solution.

As for the limits of scientific understanding, however, I have not argued that all empirical phenomena can in fact be scientifically explained. Whether that is so is an empirical question which cannot be settled by philosophical arguments a priori. For the answer depends on the nomic structure of the world. Suppose, for example, that, as assumed in contemporary physics, radioactive decay is subject to no deterministic laws, but only to probabilistic-statistical ones. Then there can be no explanation of why, in a given sample of a radioactive element with a half-life of, say, 100 years, this particular atom decays now, while that one survives for another 10,000 years. Each of the occurrences has an extremely low probability, given the relevant half-life law; neither can be explained by showing that according to that statistical law it is to be expected with high probability. ${ }^{\mathbf{1 6}}$ But then, if science could determine that the given half-life law was an ultimate law of nature for events of the kind considered, it would have said all that can be said by way of accounting for individual cases.

This kind of example, however, does not prove that the decay events referred to are "in principle" beyond the reach of scientific explanation; for to establish that claim, it would have to be proved once and for all that there are no other laws governing the events in question: and such a proof, of course, is impossible.

Finally, it should be borne in mind that we have dealt here only with problems concerning our knowledge and understanding of empirical facts. There are many deeply perplexing questions, of course, which are not of a purely empirical character; among them moral problems, to whose solution scientific inquiry can make essential contributions, but which cannot be solved on the basis of empirical knowlege alone. Human concerns that are not of a purely empirical character are sometimes voiced in the form of questions of fact, and they may then take on the appearance of absolutely unsolvable riddles. This is true, I think, of the question "Why is there anything at all and not rather nothing?" Construed literally as requesting an explanation of something from nothing, the question is incoherent. But it may well be viewed instead as expressing a deep sense of wonder at the vast and endlessly diverse and complex universe in which we find ourselves. Such wonder is not, or not exclusively, a yearning for factual knowledge; it cannot be properly expressed as an empirical problem, and thus it does

16 The general conception of probabilistic-statistical explanation which forms the background for these brief remarks is presented in Hemple, loc. cit., pp. 381-393; an example concerning radioactive decay is discussed on p. 392. 
not admit of a scientific answer.

As Wittgenstein says: "We feel that even if all possible scientific questions be answered, the problems of life have still not been touched at all. Of course there is then no question left, and just this is the answer."17

17 Ludwig Wittgenestein, Tractatus Logico-Philosophicus. London: Kegal Paul, Trench, Trubner \& Co., Ltd.; New York: Harcourt, Brace \& Company, 1933; 6.52 (italics quoted). 